\title{
El golpe de Estado argentino y su recepción por parte de la diplomacia mexicana. Itinerarios y posicionamientos político-intelectuales en los inicios del autoritarismo en la Argentina de 1930
}

\author{
Cecilia Guadalupe Neubauer*
}

Recibido el 29 de septiembre de 2018; aceptado el 11 de diciembre de 2018

\section{RESUMEN}

El golpe de Estado de 1930 y el recambio político que aconteció como su consecuencia inmediata fueron objeto de atención por parte de la representación mexicana en Buenos Aires. Los informes diplomáticos del embajador mexicano en Buenos Aires, Rafael Cabrera, recabados en el archivo histórico de la Secretaría de Relaciones Exteriores de México mostraron a la superioridad la emergencia del creciente autoritarismo por la presencia tutelar de los militares en la política local. Sumado a lo anterior, desde México se manifestaron voces contrarias a la interrupción democrática porteña, como el movimiento estudiantil latinoamericano y el sindicalista Vicente Lombardo Toledano, quienes en diferentes espacios sostuvieron la defensa de la democracia en Argentina contra el nuevo régimen. Como resultado, la década de 1930 estuvo caracterizada por el creciente distanciamiento entre ambas naciones, debido a programas políticos completamente divergentes.

Palabras clave: golpe de Estado, diplomacia, intelectuales, México, Argentina.

* Doctora en Historia, Universidad Nacional Autónoma de México (UNAM), correo electrónico: gneubauer16@gmail.com 
The argentinian coup d'état and the mexican diplomatic reception. Pollitical and intelectual Itineraries at the beginnings of authoritarianism in Argentina in 1930

\section{Abstract}

The coup d'état of 1930 and the political change that took place as its immediate consequence were the subject of attention by the Mexican representation in Buenos Aires. The diplomatic reports elevated by Rafael Cabrera, ambassador of Mexico in Argentina, seen on the Historic Archives of the Foreign Relations Secretary of Mexico, showed to the superiority the emergence of growing authoritarianism due to the tutelary presence of the military in local politics. Added to the above, from Mexico some voices were heard against the democratic interruption of Buenos Aires, such as the Latin American student movement and the trade unionist Vicente Lombardo Toledano, who in different spaces supported the defense of democracy in Argentina against the new regime. As a result, the thirties were characterized by the growing distance between the two nations, due to completely divergent political programs.

Key words: coup d'état, diplomacy, intellectuals, México, Argentina.

\section{INTRODUCCIÓN}

— n Argentina, como en otras realidades del continente, la década de 1930 Eestuvo caracterizada por las consecuencias del impacto de una crisis sistémica, ${ }^{1}$ que en su caso particular se materializó con la primera interrupción institucional de la vida política nacional con el derrocamiento del presidente Hipólito Yrigoyen de la primera magistratura de la nación y el ascenso del sector militar comandado por José Felix Uriburu al poder. Este contexto de cambios tuvo como resultado la toma de nuevos posicionamientos en la arena diplomática y representativa para los diferentes países de América Latina.

El objetivo del presente trabajo es analizar este acontecimiento y su conocimiento en México a través de fuentes diplomáticas de la embajada mexicana en Buenos Aires, para posteriormente señalar las reacciones en el arco político-intelectual mexicano, ${ }^{2}$ donde su impacto se visualizó en

1 Hablamos de crisis sistémica ya que en los años 30 la crisis fue económica, política, de valores y de hegemonía. Véase Ansaldi, "Tierra en llamas. Una introducción a América Latina en los años 1930”, pp. 13-49.

2 En cuanto a la reconstrucción de los vínculos y redes intelectuales latinoamericanas, sus influjos e influencia, algunos trabajos señeros permiten el abordaje de la presente temática: 
el repudio que el golpe de Estado argentino generó en el marco del Congreso Iberoamericano de Estudiantes y en la voz del sindicalista e intelectual Vicente Lombardo Toledano, quien había conocido el estado de situación que desencadenó la llegada de los militares al poder debido a un viaje anterior a Sudamérica, a donde se dirigió para participar en el Congreso internacional universitario, realizado en la ciudad de Montevideo durante el mes de marzo de 1931, como delegado de la Universidad de México. Anteriormente, las naciones mexicana y argentina ${ }^{3}$ vivenciaron un breve, pero intenso acercamiento durante la década de los años 1920; debido a los viajes por el continente y la diplomacia cultural, maestros y estudiantes estrecharon lazos ideológicos e intelectuales organizados bajo la égida del Reformismo universitario argentino. Con el correr de la década, y con el advenimiento del golpe de Estado, los años 1930 reconfiguraron esta relación que tuvo como consecuencia el progresivo distanciamiento político de estos países.

I.

El primer golpe de Estado en Argentina, pergeñado en septiembre de 1930 fue producto de múltiples factores. Por un lado, el impacto de la crisis económica mundial repercutió en la arena política local en el marco de un desgaste progresivo de la Unión Cívica Radical como fuerza política. Al frente de la presidencia (y de la Unión Cívica Radical) se encontraba un Hipólito Yrigoyen ya muy anciano, cuya capacidad de maniobra y negociación se vio claramente disminuida y cuestionada por otros sectores políticos, que insistían en modificar las prácticas impuestas que caracterizaban a la "política criolla” de su partido. ${ }^{4}$

Alexandra Pita, La unión latinoamericana y el boletín Renovación. Redes intelectuales y revistas culturales en la década de 1920, México, El Colegio de México, 2009; y Redes intelectuales transnacionales en América Latina durante la entreguerra, México, Porrúa 2016; Aimer Granados y Carlos Marichal, Construcción de las identidades latinoamericanas. Ensayos de historia intelectual, S. XIX y XX, México, El Colegio de México, 2009; Sebastián Rivera Mir, Militantes de la izquierda latinoamericana en México (1920-1934). Prácticas políticas, redes y conspiraciones, México, El Colegio de México-SRE, 2018.

3 En cuanto a la reconstrucción de las relaciones entre México y Sudamérica en las etapas previas al golpe de Estado, véase Yankelevich Miradas Australes, Propaganda, Cabildeo y proyección de la Revolución Mexicana en el Río de la Plata 1910-1930, México, INHERM, 1997. Daniela Spenser, En combate. La vida de Lombardo, México, Debate, 2017.

4 Se entiende por "política criolla" a un estilo de gobierno que privilegiaba la actividad fuera de los canales institucionales, con una fuerte base situada en las formaciones barriales de la Unión Cívica Radical (mejor conocidas como comités), donde el partido reclutaba a sus simpatizantes o "correligionarios", fuerza básica del desarrollo de la burocracia durante la etapa del radicalismo en el poder. En cuanto a los trabajos sobre el golpe de Estado de 1930 en Argentina y su impacto a nivel continental en cuanto a los procesos y los actores la bi- 
La década que se inauguraba ha quedado forzadamente configurada en la historia y en la cultura argentina como la década infame. Tal infamia, en breves palabras, radicó en la práctica sistemática del fraude electoral, la corrupción instalada en la burocracia estatal, así como en la profunda crisis económica que siguió al colapso económico mundial de 1929, las ollas populares como medios de subsistencia y de las consecuencias del tratado Roca-Runciman, como símbolos de la injusticia social y de la entrega del país al imperialismo inglés. ${ }^{5}$ El quiebre de aquella representación de una Argentina pujante llevó a los intelectuales a la búsqueda de las causas que expusieran las razones de lo que se visualizaba como el rotundo fracaso de un proyecto de país. ${ }^{6}$ Si bien esta etapa tiende a verse como una etapa de estancamiento y desasosiego, las investigaciones actuales afirman que se encontró marcada por una coyuntura de fortísima discusión política, gran movilización pública de escritores y ensayistas, de fundación de variadas empresas intelectuales muy activas y de intervención intensa de los partidos en el plano cultural. ${ }^{7}$

El intelectual dominicano Pedro Henríquez Ureña, quien residía en aquel momento junto a su familia en Buenos Aires, caracterizó brevemente al primer gobierno de facto en Argentina:

El gobierno provisional es de lo más “aristocrático”: la impresión más inmediata, de que el Barrio Norte le había escamoteado la revolución al pueblo, es ahora general. Pero todo el mundo lo prefiere así, al trueque de salir del "Irigoyenismo” (sic). Se espera que las elecciones lo arreglen todo. La desapari-

bliografía es vasta y diversa. Recomendamos revisar Tulio Halperín Donghi, La República imposible, Buenos Aires, Ariel, 2002; Cataruzza, Alejandro (dir.), Nueva Historia Argentina. Crisis económica, avance del Estado e Incertidumbre política (1930-1943), Buenos Aires, Sudamericana, 2001; Devoto Fernando, Nacionalismo, fascismo y tradicionalismo en la Argentina moderna, Buenos Aires, Siglo XXI, 2002; Daniel Kersffeld, Contra el imperio. Historia de la liga antiimperialista de las Américas, México, Siglo XXI, 2012; Alejandro Schenider y Pablo Pozzi, Entre el orden y la Revolución. América Latina en el siglo XX, Buenos Aires, Imago Mundi, 2004.

5 Terán, Historia de las ideas en Argentina: diez lecciones iniciales (1810-1980), p. 229. El mencionado tratado, firmado en 1933 entre Julio Argentino Roca (h) y el encargado de negocios inglés Walter Runciman, consistía en el reaseguro de la cuota de carnes exportadas a Inglaterra luego del cierre de los mercados y la preferencia de esta última por las naciones del Commonwealth debido al colapso económico de 1929. Así, Argentina se aseguraba una cuota exportable de carnes, mientras que Inglaterra se beneficiaba con la disminución de impuestos a sus bienes importados en el mercado argentino, generando una extrema dependencia de las exportaciones argentinas y una invasión de productos ingleses al puerto de Buenos Aires.

6 Ibídem.

7 Ibíd., p. 136. 
ción del radicalismo, que no era nada, deja frente a conservadores $\mathrm{y}$ socialistas. ${ }^{8}$

El golpe de Estado visibilizó nuevamente un segmento social que había sido desplazado por los radicales desde 1916: los viejos conservadores, derrotados años antes por una ley de sufragio electoral masculino, ${ }^{9}$ regresaban al poder gracias a la cercanía con la facción militar comandada en aquel momento por el general Uriburu. La interrupción democrática reflejó el estado de desgaste de la política argentina de aquel momento: una columna de cadetes y oficiales organizados partió del Colegio Militar, atravesó la ciudad y se dirigió al congreso de la Nación, donde ocurrieron los primeros enfrentamientos dejando como saldo dos cadetes muertos. Posteriormente, al llegar a la casa de gobierno, un Hipólito Yrigoyen enfermo, firmó su renuncia, convirtiéndose así en el primer presidente derrocado en la historia argentina el 6 de septiembre de 1930.

Para 1931, la situación política en Argentina reflejaba su carácter inconstitucional y de violencia creciente. La clausura de periódicos socialistas, como La Vanguardia y El Socialista Independiente, ${ }^{10}$ sumada a la intervención de la Universidad Nacional de Córdoba — cuna del reformismo—- y la expulsión de profesores y estudiantes "por actos de disciplina y subversión”, ${ }^{11}$ indicaban un paulatino viraje hacia un autoritarismo de derecha en el que la pluralidad de ideas estuvo amenazada, así como la autonomía de los claustros universitarios.

Para el mes de agosto de aquel año, las diferentes fuerzas políticas comenzaron a delinear sus pautas de acción conforme a la convocatoria a elecciones definidas para el día 8 de noviembre de $1931 .{ }^{12}$ Desde el exilio, el ex presidente Marcelo T. de Alvear ${ }^{13}$ fue proclamado por las fuerzas radicales

8 Reyes-Pedro Henríquez Ureña, Epistolario Intimo, Carta de Pedro Henríquez Ureña a Alfonso Reyes, 19 de septiembre de 1930, p. 413.

9 La sanción de la ley de sufragio electoral masculino en 1912 abrió las puertas de la contienda política de 1916 al radicalismo, desplazando al sector conservador representado por el Partido Autonomista Nacional (PAN). De aquellos comicios, resultó electo presidente Hipólito Yrigoyen, quien gobernó Argentina entre 1916 a 1922 y volvió a ocupar la primera magistratura en 1928. Como se ha mencionado, los efectos de la crisis económica de 1929 generaron su derrocamiento.

10 "Fueron cerrados dos diarios y se suspendió a otro por 48 horas", La Nación, Buenos Aires, 23 de julio de 1931.

11 “Ha expulsado el gobierno a profesores y alumnos de la Universidad de Córdoba”, La Nación, Buenos Aires, 30 de julio de 1931.

12 AHSREMEX, Sección Argentina, Informes políticos de 1931, exp. 41-7-34, 82-0/870 (512), 29 de agosto de 1931.

13 Alvear ocupó la primera magistratura Argentina entre los años 1922 a 1928, exponente del radicalismo antipersonalista, contrario a Yrigoyen. 
como candidato, mientras que el sector conservador conformó la Concordancia, una alianza de partidos entre el Partido socialista independiente, el Partido Demócrata Nacional, y la Unión Cívica Radical Antipersonalista. El candidato de esta fuerza política fue el general Agustín P. Justo, cuyo ascendente dentro del sector militar paulatinamente desplazó a Uriburu ${ }^{14}$

Para el mes de octubre, a semanas de realizarse las elecciones, una denuncia por parte del Partido Socialista acerca del secuestro de libretas de identidad, (las cuales sirven para acreditar la categoría de elector) a menos de dos semanas de los comicios presidenciales, ${ }^{15}$ puso en tela de juicio la legitimidad de los resultados, y reveló la creciente utilización que tuvo, con el correr de la época, la manipulación de las instancias electorales. Frente a este cuadro de situación, la Unión Cívica Radical se decidió por la abstención en las elecciones presidenciales. ${ }^{16}$ El sistema político argentino sufría así un golpe de legitimidad que marcaría a gran parte de la década entrante.

\section{II.}

En los informes políticos elevados por la Embajada de México en Buenos Aires a la Secretaría de Relaciones Exteriores, la primera interrupción constitucional en Argentina fue vista como una necesidad de quitar del poder a un sistema acabado. El radicalismo anteriormente gobernante fue visualizado como un desastre, en el que hordas arbitrarias cometían abusos y delitos reiterados contra la paz pública. ${ }^{17}$ Una de las críticas más frecuentes por los medios periodísticos más importantes del país tuvo que ver con la identificación del Estado con el partido, mediante la subordinación de elementos oficiales ante cualquier conveniencia electoral.

Para el $1^{\circ}$ de febrero de 1931, el poblano Rafael Cabrera fue nombrado Embajador Extraordinario y Plenipotenciario de México en Buenos Aires. ${ }^{18}$

14 Pero era el general Agustín P. Justo, jefe de otro de los grupos complicados en el golpe, quien contaba con la mayoría de las simpatías ideológicas y las lealtades entre los cuadros militares destacados. Justo, que había sido ministro del ex presidente Alvear, disponía también de un amplio sistema de relaciones con dirigentes políticos, en particular con sectores del radicalismo.

15 AHSREMEX, exp. 41-7-34, 870(510), 21 de octubre de 1931, p.1.

16 Ibíd., p.6.

17 Archivo Histórico de la Secretaría de Relaciones Exteriores México (en adelante AHSREMEX), Sección Argentina, Informes políticos de 1931, exp. 41-7-34, f. 1-3.

18 Rafael Cabrera (1884-1943). Poeta mexicano. Agregado diplomático en Bélgica y Embajador de México en la República Argentina. Culmina sus funciones el 31 de diciembre de 1934. Es reemplazado por Jaime Torres Bodet como encargado de negocios. AHSREMEX, (Legajo Representantes diplomáticos en Argentina, exp. 35-12-54. 
El embajador saliente, el escritor Alfonso Reyes, trasladado a Brasil, había dejado tras su paso una estela de vínculos culturales y literarios que posicionaron a la embajada mexicana como un faro de intercambios y redes intelectuales latinoamericanas. Con Cabrera se conservaba la tradición mexicana de la diplomacia de las letras, o el envío de intelectuales reconocidos a aquellos países con quienes México tuviera intereses significativos. Cabrera intentó mantener la mística impuesta por Reyes, heredada de la diplomacia del Carrancismo y así continuar con la tradición de considerar a la sede diplomática como la irradiación de la cultura mexicana, a través de la participación de diferentes actividades intelectuales y educativas como un modo de legitimar a la Revolución mexicana en otros lugares del continente.

Derivados de la misma situación política por la que atravesaba en ese momento la Argentina, la embajada de Rafael Cabrera observó de cerca los acontecimientos emanados del primer Golpe de Estado y sus consecuencias inmediatas. Según el embajador mexicano, Uriburu:

Se ha erigido en un árbitro de las elecciones, cuando su papel debería limitarse a convocar a elecciones (sic) y entregar dócilmente el poder en manos de las personas designadas por la voluntad popular, ya que el civismo de este país y su forma de votación reducen al mínimum los fraudes siempre posibles e inevitables. ${ }^{19}$

Para el diplomático mexicano existían dos vías para resolver este caso: por un lado, impedir el retorno de la explotación vergonzosa de la cosa pública, representado por el radicalismo derrocado y por otro lado, evitar la prolongación indefinida del gobierno de facto, que ya comenzaba a abrogarse facultades en el terreno de la batalla política que no le correspondían. ${ }^{20} \mathrm{La}$ representación mexicana siguió con mucha atención los eventos que conllevaron el regreso a la institucionalidad, la proscripción del radicalismo ${ }^{21}$ y el levantamiento armado al norte de Argentina, más precisamente en la provin-

19 AHSREMEX, Sección Argentina, exp. 41-07-34, Informes políticos de 1931, exp. 870- (510) Carta Reservada del 26 de julio de 1931.

20 AHSREMEX, Sección Argentina, exp. 41-07-34, Informes políticos 1931, exp. 870-(510). Carta reservada de Rafael Cabrera al ministro de relaciones exteriores, 22 de julio de 1931, p. 2.

21 La persecución iniciada por el gobierno provisional hacia la Unión Cívica Radical para impedir que participara en la contienda electoral de 1932 tuvo como consecuencia la decisión del partido de no presentarse a las elecciones presidenciales. El radicalismo se llamó a la abstención, que podría verse como una forma de deslegitimar el proceso electoral que definiría el restablecimiento de la democracia en Argentina. 
cia de Corrientes dirigido por el general Gregorio Pomar ${ }^{22}$, hechos que marcaron el regreso de los conservadores a la arena política argentina.

En dicho contexto, el embajador mexicano se mostró muy atento al acercamiento progresivo de los Estados Unidos hacia la Argentina. Una publicación del matutino La Prensa ${ }^{23}$ destacó la idea de avance tanto de Argentina como de Estados Unidos, frente al relativo "atraso" del resto del continente, incluida la nación mexicana. Según Rafael Cabrera, así se olvidaba:

Que hay un país fronterizo con los Estados Unidos en el que pasaron algunas cosas antes de que Argentina existiese; un país agobiado de muchas tradiciones y de pasmosas culturas autóctonas, un país en perpetua inquietud espiritual y que trata de renovarse y de superarse todos los días; que ha sido el campo de combate de las ideas de libertad más generosas; siempre a la vanguardia en el estudio y la solución de los más graves problemas políticos y sociales; que por su amor desmedido a la libertad y al bienestar humano, ha inundado de sangre su suelo repetidas veces; un país que, consciente, y orgulloso de su estirpe, $\underline{\text { se }}$ solidariza con todos sus hermanos de América, y en el lugar de más peligro, en la frontera espiritual de una raza, se endereza cuán grande es, y sin arrogancia pero con el mayor decoro, austero, tenaz, hace esfuerzos patéticos y sobrehumanos para afirmar y salvar la tradición común a todos los pueblos de la América hispana, que como un don terrible, le legó el destino. ${ }^{24}$

Frente a esta declaración de principios que realizó el embajador mexicano, interesa destacar dos elementos. Por un lado, la idea de solidaridad entre los países latinoamericanos, que según la lectura de Cabrera, esta actitud de amistad fue abandonada por Argentina, pero no así por México. Asimismo, la intromisión de Estados Unidos en Sudamérica también tuvo su manifestación concreta en la arena económica, en un intento de quebrar el cuasi monopolio inglés del comercio en el Río de la Plata. Como antesala

22 Motín militar que estalló en la ciudad de Corrientes el 20 de abril de 1931, liderado por el teniente coronel Gregorio Pomar, ex edecán del presidente depuesto Hipólito Yrigoyen. Luego de matar personalmente al jefe del $9^{\circ}$ regimiento de infantería, se declaró en abierta rebelión contra el gobierno secundado por fuerzas del mencionado regimiento y por civiles pertenecientes al Partido Radical Personalista. Intentó robar la sucursal del Banco de la Nación Argentina, cortó las comunicaciones ferroviarias inmediatamente al sur de Corrientes, voló un puente y se apoderó de la ciudad de Resistencia, capital del Chaco. Posteriormente, depusieron las armas en la localidad paraguaya de Humaitá. (...). La rebelión exterioriza el estado de alma de una parte de este pueblo por lo menos y en un estado de aumento considerable de la tensión política.

23 “Vinculación espiritual con los Estados Unidos”, La Prensa, Buenos Aires, 27 de agosto de 1931.

24 AHSREMEX, Sección Argentina, Informes políticos 1931, exp. 41-07-34-850(510), f.3. El subrayado es nuestro. 
del acuerdo Roca-Runciman firmado en 1933, el gobierno argentino comenzaba a sentir resquemores en cuanto a la implementación de una política aduanera proteccionista que afectase el comercio de los productos argentinos por parte de Gran Bretaña: “conocidas son las tendencias del actual gabinete inglés, apoyado según parece por la mayoría conservadora del Parlamento, que tienden a proteger la producción agrícola de la Gran Bretaña y de sus colonias." ${ }^{25}$ La aproximación a los Estados Unidos se profundizó con la llegada al poder del general José F. Uriburu más allá que su poder emanara de un golpe de Estado. En una entrevista éste:

afirmó que tan pronto se le presentase la primera oportunidad, demostraría los sentimientos amistosos que la Argentina tiene para con los Estados Unidos. (...) Esta actitud abierta y cordial contrasta con el espíritu hermético y reservado que caracterizó al gobierno de Irigoyen $(\mathrm{sic}) .^{26}$

En otro orden de temas, desde el mirador mexicano, las noticias provenientes de la representación diplomática argentina en México reflejaron las profundas diferencias entre el gobierno mexicano y el argentino. El golpe de Estado vino a profundizar las diferencias políticas. Durante 1930, los informes políticos que llegaban a Buenos Aires desde México documentaron con detalle la consolidación en el poder de los herederos directos de la Revolución. La transmisión de mando presidencial que tuviera como protagonista a Pascual Ortiz Rubio fue seguida muy de cerca por la legación argentina instalada en México, opacada solamente por el atentado que sufriera luego de la jura presidencial, del cual se informó que había salido ileso. ${ }^{27}$

Los informes de la representación argentina en México rebelaron paulatinamente los vaivenes de la política mexicana cooptada por los generales herederos de la Revolución. Las discrepancias entre el Partido Nacional Revolucionario (en adelante PNR) y los representantes de la Cámara de Diputados - debido al enfrentamiento entre las facciones rojas y blancas del PNR que integraban la comisión permanente del Congreso, enfrentados por obtener la mayoría en vistas a la elección de diputados y senadores- se encontraban estrechamente relacionadas con un problema de representación política en México debido a la disminución del número de escaños que iban a contender en la siguiente elección. ${ }^{28}$ La resultante de dicho conflicto con-

25 AHSREMEX, op cit., Carta reservada del 4 de diciembre de 1931.

26 “Estados Unidos y la América del Sur”, en El Universal, 3 de octubre de 1930.

27 Archivo Histórico del Ministerio de Relaciones Exteriores y Culto de Argentina (en adelante AHMRECA), División Política, Sección México, exp. 1, año 1930, Política Interna, f. 1, no. 1, p.76.

28 AHMrECA, División Política, Sección México, exp. 1, año 1930, Política Interna, f.112-119. 
sistió en la depuración de los elementos disruptivos del partido, marcados por el alejamiento de Emilio Portes Gil de la presidencia del partido, reemplazado por el general Lázaro Cárdenas, a la vez de la consolidación de Plutarco Elías Calles como árbitro de la situación política del país. ${ }^{29}$

Hasta México llegaban los informes desde el horizonte argentino acerca del atentado al derrocado presidente Hipólito Yrigoyen. En este clima, varios sectores levantaron la voz en contra del régimen instalado en Argentina por el golpe de Estado. Reunidos en la capital, entre el 17 de diciembre de 1930 al 3 de enero de 1931, en el Primer Congreso Iberoamericano de estudiantes, éstos por medio de un escrito dirigido al presidente provisional de la República Argentina, expresaron:

su protesta más enérgica por las despóticas dictaduras que ahogan la ciudadanía y la dignidad de nuestros pueblos; su adhesión inquebrantable al Maestro Palacios y a la juventud Argentina (sic) que lucha contra la dictadura arbitraria del Gral. Uriburu, solidarizándose con los ideales y la acción de la juventud revolucionaria argentina (...) que en lo sucesivo, las protestas que motiven las intervenciones gubernamentales en la Universidad, en cualquiera de nuestros países, sean manifestadas unánimemente por los estudiantes de todo Iberoamérica que sentimos como propias, las injurias que a cualquiera de nuestras universidades se haga. ${ }^{30}$

Dicho congreso, celebrado en México, con representantes de Argentina, Bolivia, Costa Rica, Cuba, España, México, Colombia, Uruguay, Santo Domingo, y Nicaragua contó con la representación de la España republicana en asuntos netamente americanos. Entre sus propuestas se destacaron el impulso a una ciudadanía universitaria, es decir, un acuerdo para que los alumnos expulsados de su país por razones políticas pudieran continuar sus estudios en otras naciones junto a la creación de la Casa del Estudiante Iberoamericano. ${ }^{31}$ Los lazos de amistad y las redes construidas por los estudiantes latinoamericanos, la devoción por Alfredo Palacios (preso por manifestarse en contra de la dictadura) y el repudio a las dictaduras latinoamericanas, así como las intervenciones a las universidades, remiten directamente al espíritu reformista de 1918, y su vigencia a nivel continental ${ }^{32}$.

29 AHMRECA, Ibíd. Nota n 20, 13 de octubre de 1930, f.5.

30 AHMrecA, División Política, México, Embajada argentina en México, Primer Congreso Iberoamericano de Estudiantes, exp. 26, f. 1-3, diciembre de 1930.

31 Biagini, Sanguinetti, "Deodoro Roca, el movimiento reformista y la integración latinoamericana” p. 483.

32 Podría considerarse uno de los coletazos finales del reformismo universitario argentino y sus influencias a nivel continental. Entrados en la década de 1930, varios procesos como el auge 
Otro detractor mexicano al régimen dictatorial argentino, pero sobre todo su política económica, fue el sindicalista Vicente Lombardo Toledano. Posteriormente a su viaje por Sudamérica —entre febrero y abril de 1931dictó una serie de conferencias organizadas entre los meses de junio y julio del mismo año, en el anfiteatro Bolívar de la Escuela Nacional Preparatoria situada en la Ciudad de México elaboró algunos juicios sobre la situación social y económica que vivía la Argentina en aquellos años. ${ }^{33}$ Entre otras cosas, el dirigente sindical manifestó:

Los aristócratas pintan un panorama falso lleno de riquezas para los que quieran ir a tomarlas. El inmigrante se ve obligado a trabajar como aparcero, haciendo contrato por un año; paga la renta por adelantado y todos los beneficios puedan [quedar] a favor del propietario de la finca. Las máquinas las proporcionan los hacendados. Tal es la Argentina sin tango, y extiende un mapa en el que señala elocuentes rótulos: atraso, analfabetismo, alcohol en una región de los Andes, crisis y miseria en la parte vecina al Uruguay y al Brasil. Inseguridad política en el centro, peonadas nómadas, criollas sin trabajo, en la provincia de Buenos Aires, latifundios en la capital, desocupación. Según la propaganda, la inmigración encuentra todo accesible. La verdad es que la desorganización es absoluta. Los italianos cultivaron la tierra porque fueron los primeros en llegar. La macrocefalia es mayor que en Montevideo. Por último, al sur, señala el feudalismo y el desierto. ${ }^{34}$

Las conferencias de Lombardo Toledano generaron respuesta por parte de Sergio García Uriburu, representante diplomático argentino, quien enviara una carta al diario El Universal, afirmando que "en la última de las conferencias aludidas se hacen apreciaciones del todo equivocadas sobre la situación de nuestro país y se emiten juicios desconsiderados e intolerables sobre su primer mandatario y digno gobierno" ${ }^{35}$ El representante argentino tuvo la oportunidad de desmentir lo dicho por Lombardo Toledano, y posteriormente éste respondió con otro artículo en las páginas del mismo periódico:

de las dictaduras y el desmembramiento del movimiento estudiantil y sus espacios representativos como la Unión Latinoamericana fueron desintegrándose paulatinamente. Véase Bergel y Martínez Mazzola, América Latina como práctica. Modos de sociabilidad intelectual de los reformistas universitarios (1918-1930), pp. 119-145.

33 Dichas disertaciones fueron reproducidas en el número 9 de la revista Futuro, en septiembre de 1934, pp. 4-23. Para analizar la actuación de Lombardo Toledano a nivel continental, véase Patricio Herrera González, "Vicente Lombardo Toledano y el Congreso Obrero Latinoamericano (1935-1938)” en Relaciones, 138, 2014, pp. 109-150.

34 Ibíd.

35 “La República Argentina, su gobierno y el Lic. Vicente Lombardo Toledano”, El Universal, Ciudad de México, 4 de julio de 1931. 


\begin{abstract}
Deseo desde luego aclarar lo siguiente: I- Al ponderar la riqueza agrícola y ganadera de la Argentina, no he hecho sino enseñar en México lo que ese gran país es en la actualidad, a pesar de todos sus gobiernos conservadores. II- Al mostrar la carta de sus problemas humanos no resueltos: latifundio, explotación etc., - que es como una radiografía de su estructura social - no contradigo la tesis anterior: sólo revelo lo que con tanto celo y mal entendido patriotismo los argentinos ricos pretenden ocultar a todo mundo y que los argentinos inteligentes y amantes de su país exhiben y comentan a diario. III. No hay contradicción, pues, en afirmar que en la Argentina podría ser todo mejor si hubiera gobiernos inteligentes, honestos y avanzados que transformaran al país de paraíso en una casta en tierra libre de la mayoría: como tampoco existe en decir que la forma cubre un fondo que se desea esconder, ni en proclamar virtudes en un pueblo que las tiene - y grandes - a pesar de la oligarquía que lo explota. $^{36}$
\end{abstract}

Resulta significativo que sólo dos años después, para 1933, el escritor argentino Ezequiel Martínez Estrada resumió algunas de estas ideas en un ensayo vertebral para la compresión de la realidad argentina de aquel entonces. $^{37}$

\title{
III.
}

Como conclusión provisoria, la atención a la realidad argentina posgolpe por parte de la representación diplomática mexicana significó la puesta de manifiesto de la crisis política desencadenada en una nación que junto a la mexicana fue considerada por aquellos años de avanzada para el resto del continente. El primer golpe de Estado reflejó sin duda la profundización del distanciamiento político entre ambas naciones. Las noticias que llegaban desde Buenos Aires a México impactaron en ciertos espacios con una histórica vinculación con los procesos rioplatenses: los colectivos estudiantiles reunidos en el congreso iberoamericano de estudiantes, y exponentes del sindicalismo mexicano y la intelectualidad se hicieron eco de la realidad política que acontecía en la Argentina que inauguraba la década de 1930 de una forma violenta y antidemocrática. Los acontecimientos que marcaron la década, como el ascenso del fascismo en Europa, primero, y la conflagración española, después, tuvo como consecuencia un cambio en la naturaleza de la diplomacia cultural que caracterizó a la nación mexicana en

36 “Contesta al Sr. Embajador de la Argentina el Lic. Lombardo Toledano”, El Universal, Ciudad de México, 9 de julio de 1931.

37 Se trata del texto “Radiografía de la Pampa”, editado por Babel, en el año 1933. 
Argentina, orientándose hacia posicionamientos y posturas que alejaron a ambas naciones definitivamente.

\section{BibLIOGRAFÍA}

Altamirano, Carlos, Historia de los intelectuales en América Latina. Tomo II: Los avatares de la "ciudad letrada" en el siglo XX, Madrid, Katz, 2008.

Ansaldi, Waldo, Tierra en llamas. América Latina en los años 1930, La Plata, Ediciones Al Margen, 2003.

Biagini, Hugo; Sanguinetti, Horacio, "Deodoro Roca, el movimiento reformista y la integración latinoamericana" en Hugo Biagini y Arturo Andrés Roig, El pensamiento alternativo en la Argentina del siglo Xx: obrerismo y justicia social (1930-1960), Buenos Aires, Biblos, 2006, pp. 481-488.

Herrera Carlos, Camarero, Hernán, El Partido Socialista en Argentina: sociedad, política e ideas a través de un siglo, Buenos Aires, Prometeo, 2005.

Cataruzza, Alejandro (dir.), Nueva Historia Argentina. Crisis económica, avance del Estado e Incertidumbre política (1930-1943), Buenos Aires, Sudamericana, 2001.

Devoto Fernando, Nacionalismo, fascismo y tradicionalismo en la Argentina moderna, Buenos Aires, Siglo XXI, 2002.

Halperín Donghi, Tulio, La república imposible (1930-1945), Buenos Aires, Ariel, 2004.

—_, Argentina y la tormenta del mundo. Ideas e ideologías entre 1930 y 1945, Buenos Aires, Siglo XXI, 2003.

Kersffeld, Daniel, Contra el imperio. Historia de la Liga Antiimperialista de las Américas, México, Siglo XXI, 2012.

Martínez Estrada, Ezequiel, Radiografía de la Pampa, Buenos Aires, Babel, 1933.

Reyes, Alfonso; Henríquez Ureña, Pedro, Epistolario Íntimo, Santo Domingo, Editorial Universidad Pedro Henríquez Ureña, 1986.

Spenser Daniela, En combate. La vida de Lombardo Toledano, México, Debate, 2018.

Terán, Oscar, Historia de las ideas en Argentina: diez lecciones iniciales (18101980), Buenos Aires, Siglo XXI, 2012.

Warley, Jorge, Vida cultural e Intelectual en la década de 1930, Buenos Aires, CEAL, 1984. 